\title{
THE PRINCIPLE OF SYMMETRY OF KINETIC COEFFICIENTS FOR MEDIA WITH FERRITE INCLUSIONS AND ITS APPLICATION TO NONRECIPROCAL BI-ANISOTROPIC COMPOSITES
}

\author{
S.V. Zagriadski and S.A. Tretyakov ${ }^{2}$ \\ ${ }^{1}$ Science Technology Center No. 407, Department of Electrical and Computer Engineering \\ Hanyang University, Haengdang-dong 17, Seongdang-Ku, Seoul, 133-791, Korea \\ Fax: +82-2-571-4656, E-mail: zagriadski@ieee.org \\ ${ }^{2}$ Radio Laboratory, Helsinki University of Technology, P.O. Box 3000, FIN-02015 HUT, Finland \\ Fax +358-9-451-2152, E-mail: sergei.tretyakov@hut.fi
}

\begin{abstract}
The principle of the symmetry of kinetic coefficients (the Onsager-Casimir principle) is formulated for bi-anisotropic media containing dissipative ferrite inclusions and metal strip elements. Electrodynamic description of bi-anisotropic particles on the base of strip-line-coupled magnetostatic wave resonators is developed and analytical closed-form expressions for the tensor polarizabilities are obtained for an arbitrary direction of the bias magnetic field and an arbitrary resonator shape. The results open a possibility to theoretically predict properties of novel nonreciprocal electrically controllable bi-anisotropic composites with exotic electromagnetic properties.
\end{abstract}

\section{INTRODUCTION}

Reciprocal bianisotropic media, in other words, media which exhibit magnetoelectric coupling effects due to first-order spatial dispersion, can be realized as composites with metal inclusions of various shapes. Nonreciprocal bianisotropic material relations (for detailed classification of bianisotropic materials see (Tretyakov et al [1])) are used to describe moving media and magnetoelectric effects in some ferromagnetic crystals.

Recently proposed artificial composite nonreciprocal bi-anisotropic materials (Kamenetskii [2]) represent ensembles of magnetized thin-ferrite-film magnetostatic wave (MS W) resonators with a partial surface metallization. It has been shown that using various shapes of conducting strips, artificial nonreciprocal materials with novel and electrically controllable properties can be realized, For example, certain configurate ions correspond to nonreciprocal Tellegen materials (Tretyakov et al [3]) or materials with the constitutive relations of moving media (Tretyakov [4]), Such devices as twist polarizers can be realized with the new media (Linden et al [5]). To design composites with desired properties, the first problem to solve is building up a model for the polarizabilities of single metal-ferrite inclusions. Next, the composite constitutive parameters can be estimated, for example using mixture rules, and the properties of the medium understood, In this presentation we will consider polarizabilities of met al-ferrite particles, establish the fundamental principle of the symmetry of kinetic coefficients for this case, and give numerical examples for the polarizabilities as functions on the frequency, ferrite parameters, and the geometry.

S.V. Zagriadski is supported by the Ministry of Education of Korea under the program BK21. 


\section{ONSAGER-CASIMIR PRINCIPLE AND THE MATERIAL RELATIONS}

A single particle which we study is schematically shown in Figure 1. Induced electric and magnetic dipole moments $\mathbf{p}_{e}$ and $\mathbf{p}_{m}$ of such artificial particles are related to the external electric and magnetic fields by linear bi-anisotropic relations

$$
\mathbf{p}_{e}=\overline{\bar{\alpha}}_{e e} \cdot \mathbf{E}+\overline{\bar{\alpha}}_{e m} \cdot \mathbf{H}, \quad \mathbf{p}_{m}=\overline{\bar{\alpha}}_{m m} \cdot \mathbf{H}+\overline{\bar{\alpha}}_{m e} \cdot \mathbf{H}
$$

where $\overline{\bar{\alpha}}_{i j}$ are the corresponding tensor polarizabilities, and $\mathbf{E}, \mathbf{H}$ are the applied high-frequency fields. Although the nature and qualitative physical picture of the magnetoelectric coupling in these elements are known (Tretyakov et al [3]), (Tretyakov [4]) and an experimental evidence of the effect has been obtained (Kamenetskii et al [6]), the electrodynamics description of the particles and the quantitative evaluation of magnetoelectric coupling till the present time has not been performed. Here we will build the corresponding model making use of the Onsager-Casimir principle.

In the standard formulation for lossless media we would have the following symmetry relation:

$$
\overline{\bar{\alpha}}_{e m}\left(\mathbf{H}_{0}\right)=-\mu_{0} \overline{\bar{\alpha}}_{m e}^{T}\left(-\mathbf{H}_{0}\right)
$$

where the external magnetizing field plays the role of the time-odd external parameter. Superscript $T$ denotes the transpose operation. We have found that for lossy ferrite inclusions we must invert the sign of the loss parameter $\Delta H_{\text {glong }}$ with that of the bias field in the right-hand side of (2). Indeed, in modeling ferrite materials, dissipation is included by a formal replacing of the real resonant frequency $\omega_{q}$, which linearly depends on the constant bias field, by a complex value $\omega_{q}+i \omega_{r}$, where the relaxation frequency $\omega_{r}$ is the loss factor which can be assumed the same for all resonator modes. This parameter is introduced in terms of the resonance linewidth $\Delta H_{0}$ as $\omega_{r}=\gamma \Delta H_{0}$. This equivalent complex value is now the external time-odd parameter which determines the nonreciprocal properties of the medium. Thus, for lossy ferrite inclusions we must invert the sign of ' $\Delta H_{0}$ along with that of the bias field in the right-hand side. Finally, we conclude that the Onsager-Casimir principle should be written as

$$
\overline{\bar{\alpha}}_{e m}\left(\mathbf{H}_{0}, \Delta H_{0}\right)=-\mu_{0} \overline{\bar{\alpha}}_{m e}^{T}\left(-\mathbf{H}_{0},-\Delta H_{0}\right)
$$

\section{EXCITATION MODEL AND NUMERICAL RESULTS}

The approach is based on the earlier obtained solutions of self-consistent electrodynamics problems of the excitation of magnetostatic waves (Zagriadski and Rezvanov [7]), and one-port (Zagriadski [8]) and two-port (Zagriadski [9]) MS W resonators. In this formulation the dipole moments of a particle induced by an external magnetic field are found through the resonator high-frequency magnetization, which is determined taking into account the "back" influence of the current in the strip on this magnetization. Neglecting this interaction results in a non-accurate determination of the polarizabilities and their resonant frequencies.

Tensor polarizabilities $\quad \overline{\bar{\alpha}}_{e m}$ and $\overline{\bar{\alpha}}_{m m}$ are calculated in the assumption that the applied electric field $\mathbf{E}=0$ in (1). Magnetization induced by a given magnetic field $\mathbf{H}$ is found as an expansion into the series of ferrite resonator eigenmodes $\mathbf{m}_{q}$ :

$$
\mathbf{M}=\sum_{q} c_{q} \mathbf{m}_{q}, \quad c_{q}=\phi_{q} \int_{V}\left(\mathbf{H}+\mathbf{h}_{q}\right) \cdot \mathbf{m}_{q}^{*} d V
$$

where $\mathbf{h}_{q}$ is the magnetic field associated with the resonator mode $q$, and $\phi_{q}$ is the resonant coefficient inversely proportional to the norm of the mode $q$, see (Zagriadski and Rezvanov [7]). Matrices $\overline{\bar{\alpha}}_{e m}$ and $\overline{\bar{\alpha}}_{m m}$ have the following form:

$$
\overline{\bar{\alpha}}_{e m}=A\left(\begin{array}{ccc}
m_{q x}^{*} & m_{q y}^{*} & m_{q z}^{*} \\
0 & 0 & 0 \\
0 & 0 & 0
\end{array}\right), \quad \overline{\bar{\alpha}}_{m m}=B\left(\begin{array}{ccc}
m_{q x} m_{q x}^{*} & m_{q x} m_{q y}^{*} & m_{q x} m_{q z}^{*} \\
m_{q y} m_{q x}^{*} & m_{q y} m_{q y}^{*} & m_{q y} m_{q z}^{*} \\
m_{q z} m_{q x}^{*} & m_{q z} m_{q y}^{*} & m_{q z} m_{q z}^{*}
\end{array}\right)
$$


Similarly, polarizabilities $\overline{\bar{\alpha}}_{e e}$ and $\overline{\bar{\alpha}}_{m e}$ are calculated in the assumption that the external highfrequency magnetic field $\mathbf{H}=0$ in (1). To find the current induced in a narrow strip by a given uniform longitudinal electric field $\mathbf{E}$, the antenna theory is used. These polarizabilities have the following structure:

$$
\overline{\bar{\alpha}}_{e e}=C\left(\begin{array}{ccc}
1 & 0 & 0 \\
0 & 0 & 0 \\
0 & 0 & 0
\end{array}\right), \quad \overline{\bar{\alpha}}_{m e}=D\left(\begin{array}{ccc}
m_{q x} & 0 & 0 \\
m_{q y} & 0 & 0 \\
m_{q z} & 0 & 0
\end{array}\right)
$$

The model results in analytical expressions for coefficients $A, B, C, D$. In terms of the scalar coefficients in the above relations, the modified Kramers-Kronig relation reads

$$
A\left(\mathbf{H}_{0}, \Delta H_{0}\right)=-\mu_{0} D\left(-\mathbf{H}_{0},-\Delta H_{0}\right)
$$

An example of the frequency dependence of the cross-polarizabilities is given in Figure 2.

\section{CONCLUSIONS}

In this paper the principle of the symmetry of kinetic coefficients (Onsager-Casimir principle) has been established for lossy and dispersive metal-ferrite inclusions. Explicit expressions were derived for tensor polarizabilities of an artificial bi-anisotropic particle on the base of an arbitrary magnetized MSW resonator with a metal strip on its surface. Numerical calculations were performed for a normally magnetized ferrite disk and the fundamental resonator mode with the uniform high-frequency magnetization. The present results validate the previous qualitative analysis of the form of the constitutive relations modelling novel nonreciprocal bi-anisotropic composites (Tretyakov et al [3] ), (Tretyakov [4]) and offer a possibility to study and design novel electrically controllable bi-anisotropic composite materials for microwave applications. These new materials can possess electromagnetic properties which are not otherwise available. For example, uniaxial Tellegen media can be realized, as well as materials whose properties simulate the constitutive relations of moving media.

\section{References}

[1] S.A. Tretyakov, A.H. Sihvola, A.A. Sochava, C.R. Simovski, Magnetoelectric interactions in bianisotropic media, J, of Electromagnetic Waves and Applications, 1998, vol. 12, pp. 481-497.

[2] E.O. Kamenetskii, On the technology of making chiral and bianisotropic waveguides for microwave propagation, Microwave and Optical Technology Letters, 1996, vol. 11, pp. 103-107.

[3] S.A. Tretyakov, A.A. Sochava, D.Ya. Khaliullin, V.V. Yatsenko, Artificial nonreciprocal uniaxial magnetoelectric composites, Microwave and Optical Technology Letters, 1997, vol. 15, pp. 260263.

[4] S.A. Tretyakov, Nonreciprocal composite with the material relations of the transparent absorbing boundary, Microwave and Optical Technology Letters, 1998, vol. 19, pp. 365-368.

[5] I.V. Linden, S.A. Tretyakov, M.I. Oksanen, Conductor-backed Tellegen slab as twist polarizer, Electronics Letters, 1992, vol. 28, pp. 281-282,

[6] E.O, Kamenetskii, I. Awai, A.K. Saha, Experimental evidence for magnetoelectric coupling in a ferromagnetic resonator with surface metallization, Microwave and Optical Technology Letters, 2000, vol. 24, pp. 56-60. 
[7] S.V. Zagriadski, A.G. Rezvanov, Self-consistent problem of the excitation of beams of magnetostatic waves, Technical Physics, (American Institute of Physics), 1998, vol. 43, pp. 963-969.

[8] S.V. Zagriadski, Design theory for magnetostatic wave filters and delay lines / Theory of one port magnetostatic wave resonators, 20th European Microwave Conference Proceedings, 1990, vol. 2, pp. $1263-1268$.

[9] S.V. Zagriadski, Theory and applications of multiport structures using magnetostatic waves, $22 \mathrm{nd}$ European Microwave Conference Proceedings, 1992, vol. 2, pp. 1363-1368.

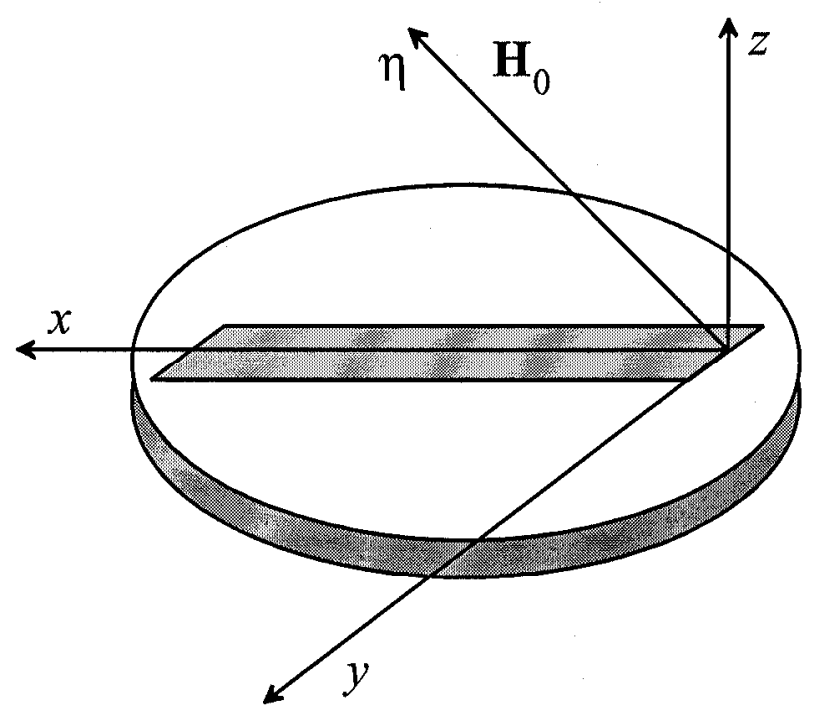

Figure 1: Geometry of an electrically controllable bi-anisotropic particle. It consists of a thin ferrite disc (MSW resonator) with a metal strip deposited on its surface. The particle is magnetized along an arbitrary direction $\boldsymbol{n}$ by constant bias field $\mathbf{H}_{0}$.
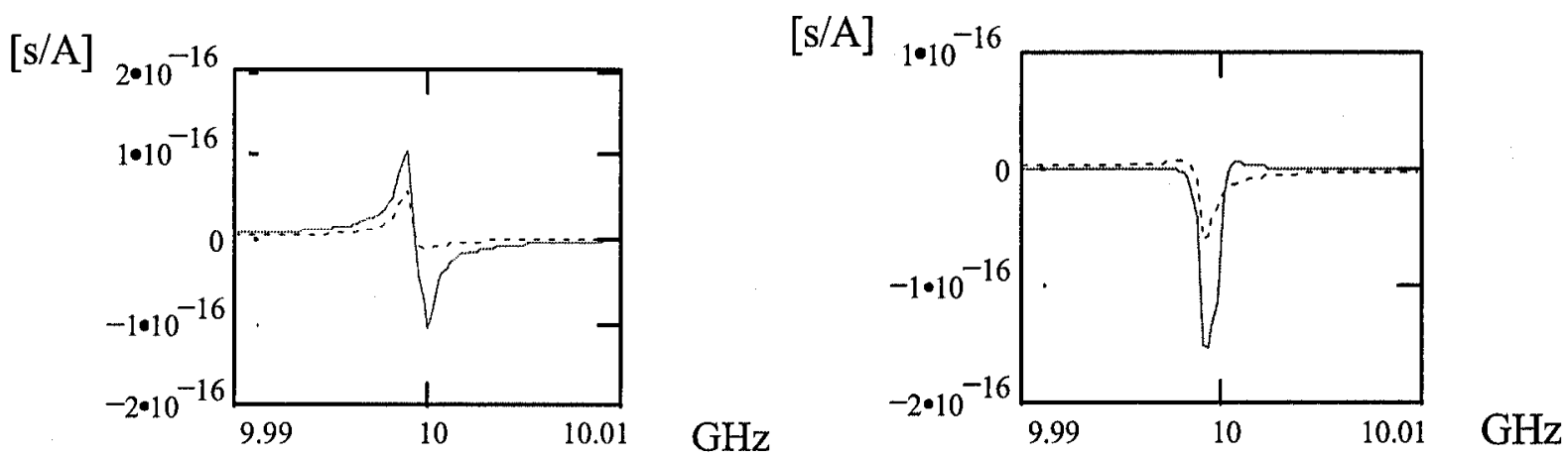

Figure 2: Frequency dependence of the real parts of the cross-polarizability coefficients. Solid curves show $-\mu_{0} \alpha_{m e}\left(-\mathbf{H}_{0},-\Delta H_{0}\right)$, and dotted curves show $\alpha_{e m}\left(\mathbf{H}_{0}, \Delta H_{0}\right)$. The common amplitude of the matrix polarizabilities is plotted. The real parts are on the left and the imaginary parts on the right. 